\section{Enzymhistochemische Untersuchungen an durstaktivierten neurosekretorischen Zellen der Ratte}

In einer vorangegangenen Mitteilung ${ }^{1}$ wurde das Enzymmuster der hypothalamischen neurosekretorischen Zentren der Ratte beschrieben und seine Entwicklung während der Ontogenese verfolgt. Seit längerem ist bekannt, dass die neurosekretorischen Zellen der Ratte durch Durstbelastung funktionellaktiviert werden können. Die Funktionssteigerung wird von erheblichen morphologischen Veränderungen begleitet ${ }^{2,3}$. Es erscheint deshalb interessant, an derartigen durstaktivierten Zellen das Verhalten einiger wichtiger Enzyme histochemisch zu untersuchen.

Dreissig erwachsenen männlichen Ratten mit einem Gewicht von 300-350 g wurde für 1-14 Tage das Trinkwasser entzogen. Von diesen erhielten 2 Tiere nach 13 Tagen Durst wieder Wasser. Die Gehirne wurden durch Medianschnitt in 2 Hälften geteilt. Eine Hälfte wurde jeweils für $24 \mathrm{~h}$ bei $4^{\circ} \mathrm{C}$ in Formol-Calcium fixiert und nach kurzer Wässerung in Aceton-Trockeneis-Gemisch eingefroren. Die andere Hälfte wurde unfixiert mit $\mathrm{CO}_{2}$ eingefroren. Aufsuchen dè Nuclei paraventricularis (N.pv.) und supraopticus (N.so.), Kryostatschnitte $14 \mu$ dick. An den Formol-Calcium-vorfixierten Schnitten wurde nachgewiesen: saure Phosphatase nach GomorI, Thiaminpyrophosphatase (TPPase) nach NovikofF' und GoldFischer 4; am unfixierten Material: Glucose-6Phosphatdehydrogenase (G-6-DH) nách Altmann und $\mathrm{CHAYEN}^{5}$, Succinodehydrogenase (SDH), Lactatdehydrogenase (LDH). In einigen Fallen wurde auch die Neurohypophyse auf G-6-DH-Aktivität untersucht.

Nach Trinkwasserentzug steigt im N.pv. und N.so. die Aktivität von G-6-DH, saurer Phosphatase und TPPase erheblich an, während $\mathrm{LDH}$ und $\mathrm{SDH}$ keine erkennbaren Veränderungen zeigen. Als erstes Ferment reagiert die G-6-DH nach 3 Dursttagen mit einem merkbaren Aktivitätsanstieg. Es kommt in erster Linie zu einer Vermehrung der auch beim Normaltier schon in geringerer Anzahl vorhandenen stark positiven Zellen. Die Aktivitätssteigerung nimmt etwa bis zum 8. Tage zu. Zu diesem Zeitpunkt ist eine fast uniforme Anfärbung beider neurosekretorischer Kerngebiete zu beobachten. Diese Reaktionsintensität bleibt bis zum 14. Dursttage konstant. Bemerkenswert ist, dass die Durstbelastung auch in der Neurohypophyse zu einer erheblichen Steigerung der G-6-DH-Aktivität führt. Bei Tieren, die $24 \mathrm{~h}$ wieder getrunken haben, hat sich die G-6-DH-Reaktion bereits wieder völlig normalisiert.

Bei der sauren Phosphatase und TPPase kommt es erst nach 5-6 Dursttagen zu einem deutlichen Aktivitätsanstieg. $\mathrm{Zu}$ diesem Zeitpunkt sind ganz auffällig 2 Typen von Zellen zu unterscheiden. Typ I: kleine kompakte Zellen mit stark TPPase-positiven, konzentrisch um den Kern geschichteten Golgi-Lamellen. Ebenfalls unmittelbar perinukleär sieht man zahlreiche, dicht gepackte saure-Phosphatase-positive Granula (vermutlich Lysosomen), die von hier in die Peripherie des Perikaryons hineinzudiffundieren scheinen. Die äusseren Anteile des Perikaryons selbst sind frei von TPPase und saurer Phosphatase. Typ II: grosse Zellen mit geschwollenem Kern und Nukleolus sowie vakuoligem Cytoplasma. Der Golgi-Apparat ist in Form von Schollen und Granula über das ganze Perikaryon verteilt und wenig TPPase-positiv. Bei der Reaktion auf saure Phosphatase finden sich nur wenige Lysosomen ohne bevorzugte Anordnung. Bei forciertem Wasserentzug werden diese Unterschiede noch deutlicher. Die Lysosomendichte in den Typ I-Zellen steigt immer mehr an, die Typ II-Zellen zeigen einen zunehmenden Zerfall des Golgi-Apparates. Nach $24 \mathrm{~h}$ Wasseraufnahme sind die Unterschiede verschwunden. Typ II-Zellen sind nicht mehr zu beobachten. Es kommt zu einer deutlichen Reorganisation des Golgi-Apparates. Die Aktivität der sauren Phosphatase ist auch 48 h nach Wasseraufnahme noch erheblich höher als beim Normaltier. Eindeutige Unterschiede zwischen N.pv, und N.so. konnten weder in der Intensität der Fermentreaktionen noch in der Geschwindigkeit des Ansprechens auf die Versuchsbedingungen beobachtet werden.

Zweierlei Zelltypen in den neurosekretorischen Kerngebieten der Ratte sind bereits lichtmikroskopisch von BACHRACH ${ }^{3}$ und elektronenmikroskopisch von $Z_{A M B R A N O}$ und DE ROBERTIS ${ }^{6}$ beschrieben worden. Die hier histochemisch charakterisierten Zelltypen scheinen mit den von diesen Autoren beschriebenen weitgehend identisch zu sein. Auf Grund allgemeiner cytologischer Kriterien wird angenommen, dass unsere Typ II-Zellen eine sekretorisch besonders aktive Funktionsform darstellen. Dazu stehen die histochemischen Befunde insofern im Widerspruch, als nicht die Typ II-, sondern die Typ I-Zellen sich durch einen hochaktiven Golgi-Apparat und intensive saure-Phosphatase-Aktivität auszeichnen. Es scheint als ob in diesen Zellen die Hauptaufgabe des GolgiApparates in der Produktion von Lysosomen, und weniger in der Sekretbereitung zu suchen ist. Hohe Sekretionsleistung und Lysosomenproduktion misssen offenbar nicht unbedingt miteinander verknüpft sein.

Die für die erhöhte Leistung zusätziich benötigte Energie beziehen beide Zelltypen offenbar über den Pentose-Phosphat-Zyklus, denn die hier untersuchten Enzyme der Glycolyse und des Zitronensäure-Zyklus zeigen keine merkliche Aktivitätsänderung. In Ubbereinstimmung damit stehen die Befunde von JoNGKIND", der mit biochemischer Methodik ebenfalls eine Aktivitätserhöhung der G-6-DH im N.so. der Ratte unter Durstbelastung nachweisen konnte.

Summary. In hypothalamic neurosecretory nuclei of rats subject to water withdrawal for 1-14 days, a marked increase in the activities of histochemically detected acid phosphatase, thiamine pyrophosphatase and glucose-6phosphate dehydrogenase is observed. LDH and SDH activities do not change. Two different cell types can be distinguished by means of the acid phosphatase and TPPase reactions.

Ch. Pilgrim

Anatomisches Institut der Universität $W$ ürzburg

(Deutschland), 11. September 1967.

1 Ch. Pilgrim, Histochemie 10, 44 (1967).

2 R. Ortmann, Z. Zellforsch. mikrosk. Anat. 36, 92 (1951).

s D. Bachrach, Z.Zellforsch. mikrosk. Anat. 47, 147 (1957).

4 A. B. Novikoff and S. Goldfischer, Proc. natn Acad. Sci. USA 47,802 (1961).

5 F. P. Altmann and J. Chayen, J1. R. microsc. Soc. 85, 175 (1965).

B D. Zambrano and E. DE Robertis, Z. Zellforsch. mikrosk. Anat. $73,414(1966)$.

7 J. F. Jongkind, J. Histochem. Cytochem. 15, 394 (1967). 